\title{
A repetitive spontaneous distal tibial insufficiency fracture: an unusual case report
}

\author{
Ebru Aytekin, ${ }^{1}$ Emrah Kovalak ${ }^{2}$ \\ ${ }^{1}$ Department of Physical Medicine and Rehabilitation, İstanbul Training and Research Hospital, İstanbul, Turkey \\ ${ }^{2}$ Department of Department of Orthopedics and Traumatology, İstanbul Training and Research Hospital, Istanbul, Turkey \\ Received: July 2015 Accepted: October 2015
}

\begin{abstract}
Stress fractures typically occur, when cyclic loading to the healthy bone exceeds the normal carrying range capacity. These fractures are most commonly seen in athletes and military recruits who are engaged with intensive physical exercise in their daily lives. Stress fractures can also occur in a variety of conditions, in which the mineral content or the elasticity of bone is abnormal and defined as an insufficiency fracture. The tibia is the most frequent location for stress fractures, while bilateral tibial fractures are extremely rare. Herein, we report a repetitive spontaneous distal tibial insufficiency fracture in the right and left distal tibia and discuss predisposing factors, diagnosis, and treatment in the light of the current literature.
\end{abstract}

Keywords: Insufficiency fracture; radiological evaluation; stress fracture; tibia.

Stress fractures (SFs) usually occur in the weightbearing bones of the lower extremity, primarily sacrum (29.6\%), tibia (23.6\%), femur neck (9.9\%), tarsal navicular (17.6\%), metatarsal bones (16.2\%) and calcaneus $(2.8 \%)$, and tend to be unilateral..$^{[1,2]}$ These fractures are most commonly seen in athletes and military recruits due to submaximal chronic overload. ${ }^{[1-3]}$ The exact phenomenon responsible for initiating a SF is still unclear. ${ }^{[3]}$ However, predisposing factors under normal stress include genetic factors, hormonal imbalances, metabolic bone disorders (i.e., osteoporosis, osteomalacia), liver and kidney failure, systemic diseases, rheumatic diseases, nutritional deficiencies, heavy smoking, and the use of medications which may cause osteoporosis. ${ }^{[3-5]}$ Herein, we report a repetitive spontaneous distal tibial insufficiency fracture in the right and left distal tibia and discuss predisposing factors, diagnosis, and treatment in the light of the current literature.

\section{CASE REPORT}

A 65-year-old male presented to the Orthopedics and Traumatology outpatient clinic with a month history of painful left ankle swelling and inability to walk. His medical history revealed no trauma. He was an ex-smoker with a four-pack-year history of smoking with intensive alcohol consumption for four days per week. Currently, he was not using cigarette or alcohol for five years. He was on an inhaled steroid due to chronic obstructive pulmonary disease for 10 years. He had the same complaints in his right distal cruris a year ago, and cas treatment was applied for eight weeks. His family history was non-specific. A written informed consent was obtained from the patient.

The patient was admitted to the Orthopedics and Traumatology outpatient clinic, as he suffered from same complaints 15 days prior. His physical examination revealed normal findings. A plain radiograph of his left cruris revealed no osseous pathology (Figure 1). Medical treatment with a non-steroidal anti-inflammatory drug was prescribed and he was scheduled for a follow-up visit within 15 days. At the follow-up visit, he had an antalgic gait pattern. There were edema and erythema at the left distal cruris. His left ankle was tender on palpation and the range of motions in all planes was

Corresponding author: Ebru Aytekin, MD. İstanbul Eŭitim ve Araştırma Hastanesi, Fiziksel Tıp ve Rehabilitasyon Kliniği, 34098 Fatih, İstanbul, Turkey. e-mail: ebruaytekin@hotmail.com 
restricted. Left cruris plain radiographs were repeated and transverse fracture line was seen in the distal tibia (Figure 2). His previous radiograph showed an improved right distal tibial fracture line (Figure 3). However, currently, he was walking in pain and the cast immobilization was planned and follow-up was recommended with a 10-day interval for the first three weeks, and 20-day interval, thereafter. During the last 20 days, the fracture was managed with a patellar tendon-bearing walking cast. The treatment was discontinued, when the callus formation was seen at least three cortices at 10 weeks. Routine laboratory test results including kidney and liver functions, calcium, phosphorus, alkaline phosphatase, and parathyroid hormone were normal. In addition, acute phase response proteins (i.e., C-reactive protein, erythrocyte sedimentation rate), rheumatic profile, thyroid functions, gonadal hormones, and tumor markers were normal. The level of $25(\mathrm{OH}) \mathrm{D}$ vitamin was $9,915 \mathrm{ng} / \mathrm{mL}$. A dual energy X-ray absorptiometry scan revealed osteopenia (Left femur neck T score: -1.5 , $\mathrm{Z}$ score: -0.5 , lumbar total $\mathrm{T}$ score: $-1.7, \mathrm{Z}$ score: -0.9 ). The patient, then, underwent magnetic resonance imaging (MRI) to exclude pathological fractures and soft tissue pathologies. There was no pathological finding except a non-displaced fracture in the distal tibial metaphyseal region (Figure 4). The bone isotope scan confirmed an increased distribution of the left

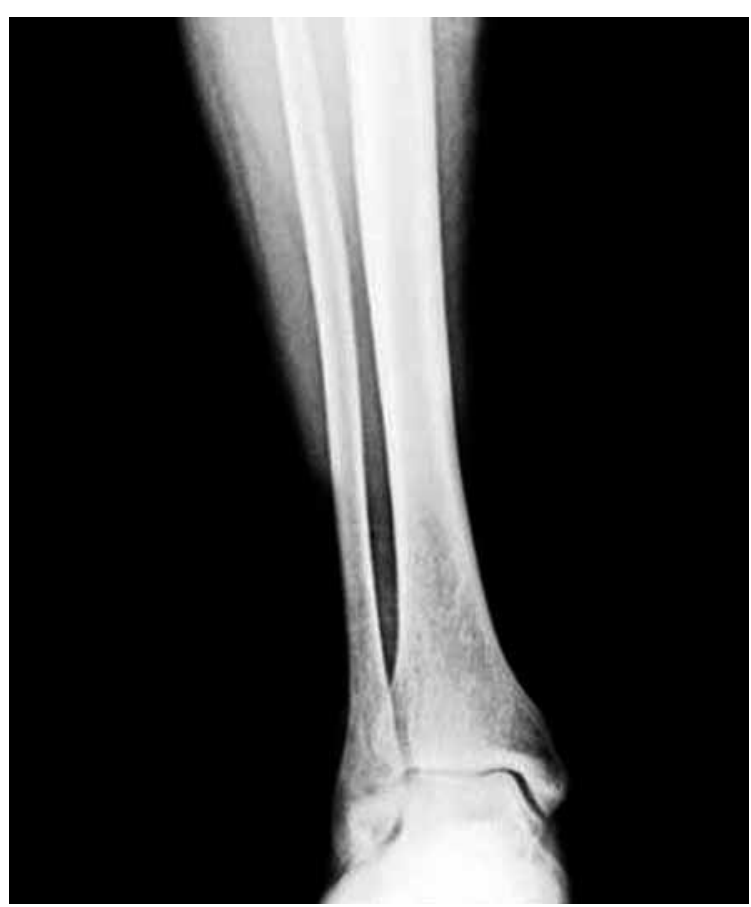

Figure 1. An undetected distal tibial fracture line in the anteroposterior radiograph of the left cruris. distal tibial metaphyseal fracture (Figure 5). Oral 300,000 IU/mL vitamin D3 (three doses, with intervals of 15 days) was prescribed. In addition, $600 \mathrm{mg}$ calcium and 440 IU vitamin D3 were given daily. During follow-up, 25(OH)D vitamin level increased up to $62,764 \mathrm{ng} / \mathrm{mL}$. At three months, alendronate $70 \mathrm{mg}$ plus cholecalciferol 2,800 IU/week was added to the treatment. Throughout the medical treatment, the range of motion, stretching and strengthening exercises were instructed. In addition, he was consulted to the department of chest diseases to change his inhaled treatment. The patient is still under follow-up in our outpatient osteoporosis clinic.

\section{DISCUSSION}

Stress fractures occur when cyclic loading to the healthy bone exceeds the normal carrying range capacity. ${ }^{[2]}$ These fractures most commonly affect athletes and military recruits who are engaged with intensive physical exercise in their daily lives. ${ }^{[1-3]}$ Two types of SFs have been described according to etiology: fatigue fractures and insufficiency fractures. ${ }^{[5]}$ A fatigue fracture occurs, when the healthy bone is exposed to abnormal muscular stress or torque. Many conditions leading an abnormal bone mineral content or elasticity such as osteoporosis, fibrous dysplasia, Paget's disease, rheumatoid arthritis, systemic lupus

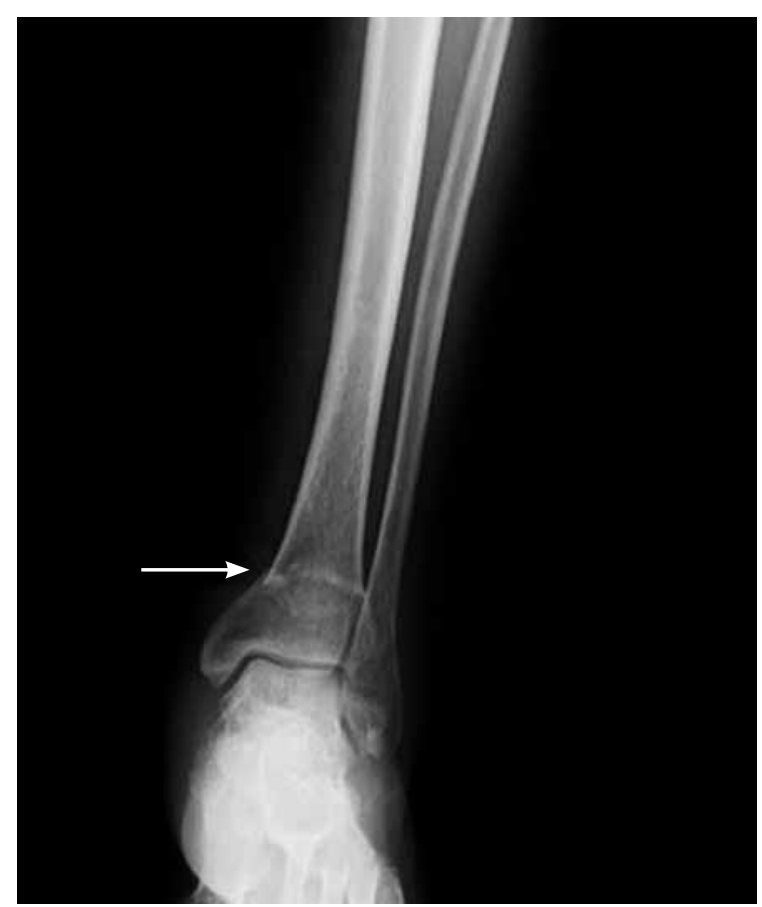

Figure 2. A transverse fracture line in the distal tibia in the anteroposterior left cruris plain radiographs. 


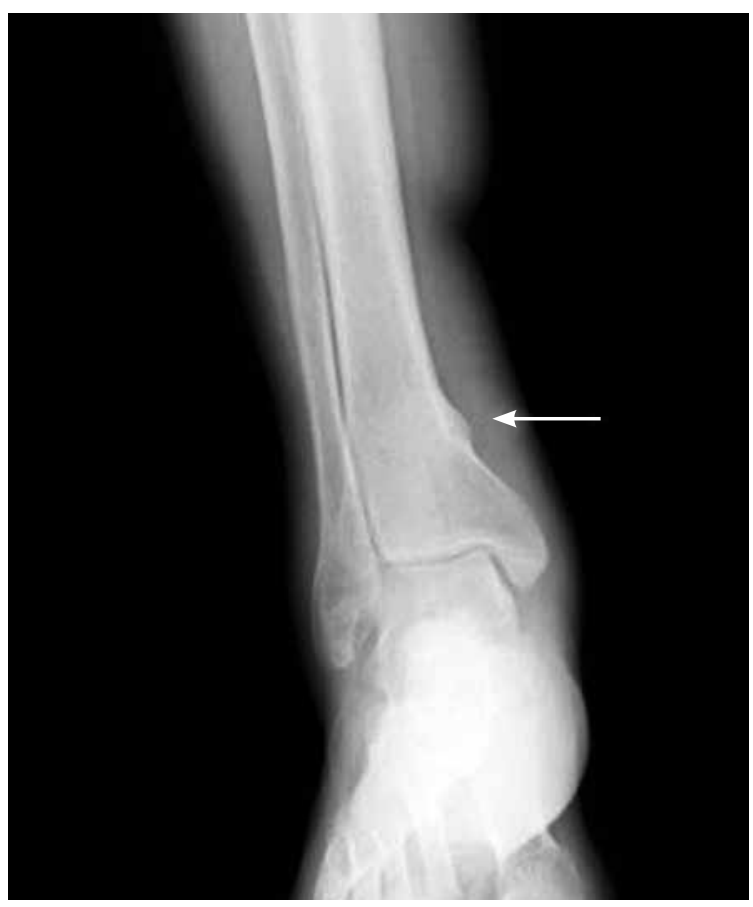

Figure 3. An anterior-posterior radiograph of right cruris showing an improved right distal tibial fracture line.

erythematosus, calcium pyrophosphate deposition and, diabetes mellitus may lead to the formation of insufficiency fractures. In addition, heavy smoking, inhaled steroid use, and amenorrhea are

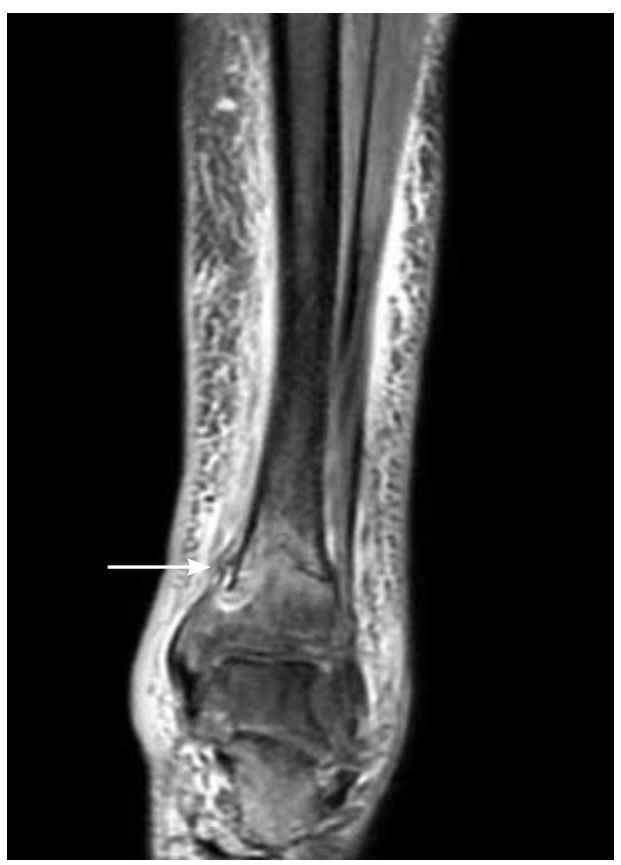

Figure 4. A coronal fat-sat magnetic resonance imaging of the left cruris showing a non-displaced fracture line in the distal tibial metaphyseal region.

the other predisposing factors. ${ }^{[3-5]}$ Our case was working as a guard, before he got retired and he had no predisposing condition which would cause overloading of the distal tibia. However, osteopenia,

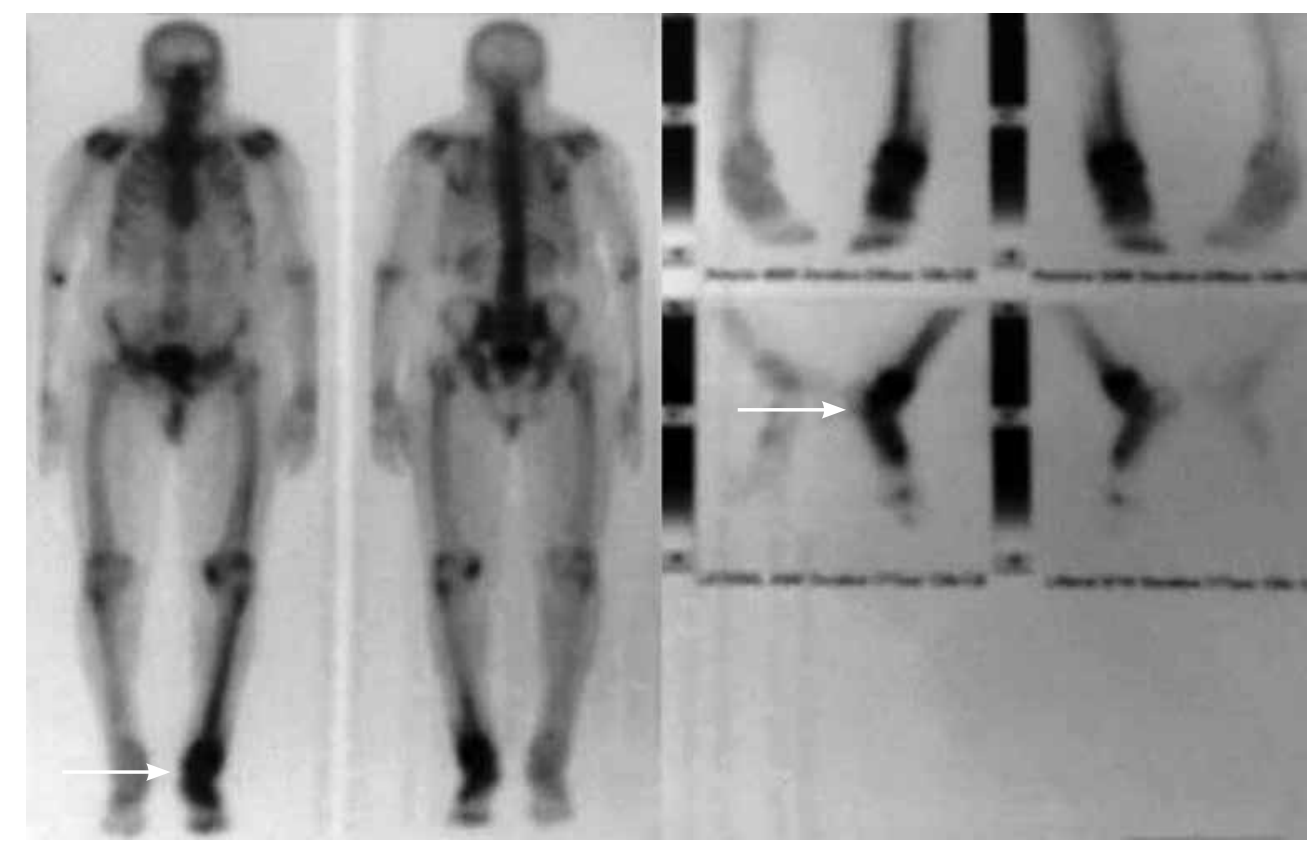

Figure 5. A bone isotope scan showing a focal increased uptake on the left distal tibial metaphyseal region. 
vitamin D deficiency, the use of inhaled steroids, and heavy smoking may have led to a spontaneous distal tibial insufficiency fracture.

Plain radiographs are not sensitive in diagnosing SFs. They are usually normal in the beginning of the disease. ${ }^{[2]}$ Since plain radiographic findings may not be obvious initially, the delay from onset to diagnosis ranges from 15 days to 18 months. ${ }^{[5]}$ Chronic stress leads to periosteal reaction and cortical thickening at the injury site, which make the fracture visible on the plain radiographs over time. A positive bone scan shows an increased uptake at the affected area. ${ }^{[2,5]}$ Similarly, in our case, plain radiography showed typical course of the fracture and the diagnosis was able to be made on Day 15.

In addition, differential diagnosis should include periostitis, medial tibial stress syndrome, tibial stress reaction, anterior compartment syndrome, infections, tumors (i.e., osteogenic sarcoma, Ewing's sarcoma, and osteoid osteoma) and metastases. Radiographs, bone isotope scanning, and MRI are necessary in the differential diagnosis. ${ }^{[2,5]}$ Radiologically, there was no evidence of malignancy in our case.

The treatment of SFs consists of primarily rest, activity modification, and cast immobilization along 6 to 12 weeks. $^{[5]}$ The majority of SFs can be effectively managed with relative rest and non-invasive interventions. ${ }^{[2]}$ Surgery with intramedullary nailing can be considered in selected cases with persistent or recurrent fractures. Furthermore, it is necessary to treat osteoporosis to prevent future hip and vertebral compression fractures. ${ }^{[5]}$

In conclusion, in the management of stress or insufficiency fractures, metabolic and systemic risk factors should be detailed and osteoporosis treatment should be started immediately to prevent the formation of new fractures and to minimize the risk.

\section{Declaration of conflicting interests}

The authors declared no conflicts of interest with respect to the authorship and/or publication of this article.

\section{Funding}

The authors received no financial support for the research and/or authorship of this article.

\section{REFERENCES}

1. Khy V, Wyssa B, Bianchi S. Bilateral stress fracture of the tibia diagnosed by ultrasound. A case report. J Ultrasound 2012;15:130-4.

2. Malkoc M, Korkmaz O, Ormeci T, Oltulu I, Isyar M, Mahirogulları M. An unusual stress fracture: Bilateral posterior longitudinal stress fracture of tibia. Int J Surg Case Rep 2014;5:500-4.

3. Tsuchie H, Okada K, Nagasawa H, Chida S, Shimada Y. Bilateral stress fracture of the fibulae and periostitis of the tibiae. Med Princ Pract 2010;19:490-2.

4. Dreher R, Buttgereit F, Demary W, Görtz B, Hein G, Kern P, et al. Insufficiency fractures in rheumatology. Case report and overview. Z Rheumatol 2006;65:417-23. [Abstract]

5. Defoort S, Mertens P. Multiple tibial insufficiency fractures in the same tibia: a case report. Geriatr Orthop Surg Rehabil 2011;2:69-72. 